\title{
Catheter-Related Infections Rate at Two University Affiliated Hospitals in the North-West of IRAN
}

\author{
Manizheh Jozpanahi ${ }^{1}$, Afsaneh Karami' ${ }^{2}$ Abdolreza Esmaeilzadeh ${ }^{3}$, \\ Samira Hanafi ${ }^{4}$, Ahmad Reza Mobaien ${ }^{5}$ and Parvin Shiri Ghidari ${ }^{6}$
}

${ }^{1}$ Department of Infectious Diseases, Zanjan University of Medical Sciences, Zanjan, Iran. ${ }^{2}$ Faculty member, Department of Infectious Diseases, Faculty of Zanjan University of Medical Sciences, Zanjan, Iran.

${ }^{3}$ Department of Microbiology and Immunology, Zanjan University of Medical Sciences, , Zanjan, Iran.

${ }^{4}$ General Practitioner, Zanjan University of Medical Sciences, , Zanjan, Iran.

${ }^{5}$ Department of Infectious Diseases, Zanjan University of Medical Sciences, Zanjan, Iran.

${ }^{6}$ Department of Nursing, Zanjan University of Medical Sciences, Zanjan, Iran.

http://dx.doi.org/10.13005/bbra/2037

(Received: 30 January 2016; accepted: 20 February 2016)

Evaluation of peripheral venous catheter culture positive rate in patients who were hospitalized on internal, cardiac, neurologic, infectious, ICU, CCU wards atBeheshti\& Vali-e-asr Hospitals in Zanjan, Iran. The relentless progress of medical science and technology has been accompanied by the development of a host of new diagnostic and therapeutic devices, each of which is associated with its own complications. One of these devices is venous catheter which unfortunately can introduce infection to the bloodstream, and as a consequence of their increasing use, bloodstream infections resulting from intravascular catheters have become a costly complication of health care (22). The rate of catheter associated bacteremia rose from 20 episodes per 1000 admissions in 1986 to 50 episodes per 1000 admission in 1993. For this reason we decided to evaluate the rate of positive cultures of peripheral venous catheters and causative pathogens and it's antibiogram in this study. In this descriptive study, after 48-72 h of intravenous catheter placement, 500 blood samples were obtained from the junctional site of peripheral venous catheter.Patients were selected randomly from internal, cardiac, neurology, infectious, ICU, CCU wards at Beheshti and vali-e-asr Hospitals. Then these samples were inoculated in blood agar. The antibiogram was performed for each causative pathogen if culture was positive. The collected data was analyzed by central and scattered index and Chi-Square test, $\mathrm{T}$ test and Mann-Whitney test. During this study, peripheral venous catheters of 500 patients [254(50.8\%) women and $246(49.2 \%)$ man] were cultured. In thirty patients [12 $(4.8 \%)$ women and $18(7.3 \%)$ men], blood culture became positive. There was no significant statistical difference in the rate of positive catheter blood culture among the different genders, wards and hospitals. Culture were positive for staphylococcus ( 29 cases) including Staphylococcus aureus in 17 cases and Coagulase negative Staphylococcus in 12 cases, and only one case was positive for gram negative rod $(E$. coli). Positive cultured organisms had the highest sensitivity to ciprofloxacin and cephalexin. Among eleven patients who received chemotherapy, three cases $(27 \%)$ had positive blood culture results $(p=0.003)$. Catheter related blood stream infection is more frequent at Beheshti and vali-e-asr Hospitals compared to the national rate of catheter related blood stream infection and we need more investigation to find out whether it is patient, catheter or operator related issue.According to the finding of this study,chemotherapic drugs usage is arisk factor for positive culture of catheter.

Key words: Catheter-related blood stream infection, chemotherapy drugs and infection, peripheral venous catheters

\footnotetext{
* To whom all correspondence should be addressed.
} 
Despite so many advances in science, technology and therapeutic tools, there are still some unwanted events that might somehow change the course of admission to the hospital. Catheter related blood stream infection is one of those disadvantages that needs to be considered seriously.

continuing with the progress of science and technology and extensive diagnostic and therapeutic tools, there are unwanted side effects alongside numerous advantages associated.

Up until recently, over 250000 CRBSIs occurred every year in the United States and over 80000 of these appeared in ICUs. These infections are associated with increased length of hospital stay from 10 to 20 days and increases in the cost of care from $\$ 4000$ to $\$ 56000^{22}$.

Intravascular device insertion can be followed by local and systemic infections. Any injection is not without a risk; even distilled liquid could be a source for an infection with Burkholderiacepacia (Pseudomonas spp) ${ }^{4}$. The risk factors for catheter related infection, include prolonged duration of usage, a history of previous catheter-related bacteremia, recent surgery, diabetes mellitus, iron overload, Staphylococcus aureus nasal colonization, old age, and low hemoglobin and serum albumin levels $7,1,20$. Determining risk factors of catheter associated infections may aid infection prevention and subsequently lower therapeutic cost and improve patient survival and quality of life ${ }^{2}$. In intravenous nutritional fluid, growth of some microorganisms has been observed ${ }^{6}$. Hydrolyzed casein solution can cause the growth of many bacteria and fungi ${ }^{10}$. Contamination of catheter tube junction with the injection sources has been identified as a focus of catheter-induced bacteremia that often caused by coagulase negative Staphylococcus ${ }^{16}$. Sepsis is the second most common cause of death in hemodialysis patients after cardiovascular disease $\mathrm{e}^{5}$. The use of antibiotic lock in addition to parenteral antibiotic therapy has been shown to reduce the relapse rate of Catheter Related Infection (CRI) in a small randomized study ${ }^{15}$. Systemic prophylactic antibiotic treatment prior to insertion of the catheter does not result in a significant reduction of $\mathrm{CRI}^{8,21}$. Catheter-related infection is associated with increasing the incidence of mortality and morbidity and due to lack of local signs of inflammation in somecases, clinical diagnosis of catheter related blood stream infection is often difficult.

\section{MATERIALS \& METHODS}

This study was done on all patients who were admitted to internal medicine, cardiology, neurology, infectious diseases wards, ICU and CCU in Zanjan University Affiliated Hospitals. After 48 hours of catheter insertion, the junction of the catheter to serum set was separated with a moist sterile swab dipped in distilled water. Then, it was rolling on the junction until enough samples obtained for culture. Then swab was placed into the broth culture media and transported to the laboratory. So, each sample entered in the culture media and after 24 hours was transferred to the specific media. Then, based on the type of microorganisms grown, organisms were transferred to Mueller Hinton medium for antibiogram . Results were reported as susceptible, semi susceptible or resistant. Simultaneous sampling, information about patients and catheters with specific codes for each were recorded in the questionnaire. Obtained information from patients and catheter characteristics, along with data collected from laboratory were analyzed by using central and dispersion parameters and Chi square, T-test and Mann-Whitney test with SPSS. Sampling was conducted according to ethics committee compliance.

\section{RESULTS}

In this study, peripheral venous cathetersof 500 patients [254(50.8\%) women and $24649.2 \%$ ) man] were cultured. Thirty patients [12 (4.8\%) women and 18 (7.3\%) men] had positive blood culture results. There was not significant statistical association between the rate of positive blood culture results and gender. 31(6.2\%) patients were in the infectious diseases ward, 164 (32.8\%) in cardiology, 171 (34.2\%) in internal medicine, 78 (15.6\%) in the neurology, 13 (2.6\%) in ICU and 43 (8.6\%) in CCU. There was no significant statistical difference in the rate of positive catheter blood culture results and the location of patients. Among culture positive group, the mean age was 54.03 23.56 and $59.47 \pm 24.17$ in culture negative group.? 
Considering different hospitals, 8 of 116 patients(6.9\%) became culture positive at Beheshti Hospital and 22 of 384 patients (5.7\%) showed positive blood cultures at Vali-e-Asr Hospital. We didn't find significant statistical difference in culture positive rate between two hospitals.

44 patients had infectious diseases, among which only two cases $(4.5 \%)$ showed positive catheter culture results. Among 204 patients who had cardiac diseases, 15(7.4\%) cases had positive blood culture results. Eighty-nine patients had an underlying neurologic disease and $9(10.1 \%)$ cases were culture positive. Of 49 patients who had respiratory diseases and 19 patients who had hematological diseases there was no positive culture. 2 of 24 (8.3\%) patients with underlying oncologic diseases were culture positive. In 43 patients with gastrointestinal diseases and 13 patients withrheumatology diseases, only one became culture positive in each group. There was no positive culture in 19 patients with nephrology problem, 9 patients with endocrine disorders, 49 patients with respiratory disease and 19 patients with hematologic problem. There weren't observed a significant relationship between related disorders and the rate of positive catheter blood culture results in both groups. 78 of 500 patients were receiving corticosteroids, among which 4 patients were culture positive. Among our study group, 154 were on antibiotic and among them 9 patients showed positive blood culture results due to catheter with no statistical significance.

11 patients were receiving chemotherapy (table 1), and 3(27\%) cases had positive catheter blood culture results $(\mathrm{p}=0.003)$. This means that chemotherapy can be a risk factor for catheter culture positive. We didn't find statistical association between the rate of positive culture and the catheter diameter.Presence of symptoms of inflammatory wasn't role in the rate of catheter cultures positive. Overall, 30 patients had positive cultures; staphylococcus (29 cases),

Table 1. Comparison of culture positive rate in 3 different groups of patients based on received medication(corticosteroid vs chemotherapeutic agent vs antibiotic)

\begin{tabular}{llccc}
\hline Medication Culture & Result & Corticosteroid & Chemotherapy agent & Antibiotic \\
\hline Consumed & Positive & $4(5.1)$ & $3(27.3)$ & $9(5.8)$ \\
& Negative & $74(94.9)$ & $8(72.7)$ & $145(94.2)$ \\
Un consumed & Positive & $26(6.2)$ & $27(5.5)$ & $21(6.1)$ \\
& Negative & $396(93.8)$ & $462(94.5)$ & $325(93.9)$ \\
Total number & & 500 & 500 & 500 \\
P-value & 0.724 & 0.003 & 0.922 & \\
\hline
\end{tabular}

*Numbers in parentheses indicate percentage

Table 2. Antibiogram results of bloodculture positive organisms

\begin{tabular}{lcccc}
\hline Antibiotic name & Sensitive & Semi sensitive & Resistant & Unknown \\
\hline Ceftizoxime & $8(26.7) \mathrm{m}$ & $6(20)$ & $14(46.7)$ & $2(6.7)$ \\
Ciprofloxacin & $10(33.3)$ & $14(46.7)$ & $6(20)$ & 0 \\
Cephalexin & $10(33.3)$ & $9(30)$ & $10(33)$ & $1(3.3)$ \\
Gentamicin & $7(23.3)$ & $22(73.3)$ & $1(3.3)$ & 0 \\
Co-trimoxazole & $4(13.3)$ & $5(16.7)$ & $21(70)$ & \\
Amikacin & $25(83.3)$ & $4(13.3)$ & $1(3.3)$ & 0 \\
Ceftriaxon & $9(30)$ & $9(30)$ & $11(7.36)$ & $1(3.3)$ \\
Cefazolin & $9(30)$ & $3(10)$ & $6(20)$ & $12(40)$ \\
Ampicillin & 0 & $13(43.3)$ & $16(53.3)$ & $1(3.3)$ \\
Tetracycline & $9(30)$ & $11(36.7)$ & $7(23.3)$ & $3(10)$ \\
Penicillin & 0 & $11(36.7)$ & $18(60)$ & $1(3.3)$ \\
\hline
\end{tabular}

*Numbers in parentheses indicate percentage 
Staphylococcus aureus (17 cases), and Coagulase negative Staphylococcus (12 cases), and one gram negative bacilli (E. coli).

Organisms isolated from positive catheter culture results had the highest sensitivity to ciprofloxacin and cephalexin. There was no sensitivity to ampicillin and penicillin, and the highest resistance was to trimethoprimsulfamethoxazole. The antibiogram result is shown in table 2.

\section{DISCUSSION}

Based on the results of this study, the rate of intravenous catheter induced positive blood culturein our 2 hospitals is $6 \%$ which is close to the rate was shown byOncu S. et al., ${ }^{13}$ on the 300 central venous catheters in 2003, and the rate of positive catheter blood culture results was 5.6\% reportedly.that was not much difference between that and our study. We know they studied the rate of infection on central venous catheter that probably will be kept longer than a peripheral venous catheter so will have some impact on the incidence rate but we are far from the almost recent data was published by $\mathrm{H}$. Shah and et in 2013 that showed the rate of blood stream infection due to venous catheter placement is $0.1 \%$. Therefore, we need more investigation to find out our higher rate of infection is patient, catheter or operator related.

There are also other studies like Band J. and et al. ${ }^{3}$ and Pujol M. et al. ${ }^{14}$ that reported the frequency of positive catheter blood culture results was $4 \%$ and $4.2 \%$ respectively, which is less than present study.

Thirty positive catheter blood culture results obtained during this study. 12 cases were females (40\%) and 18 cases were males (60\%). We didn't find a significant statistical relationship between gender and the rate of infection which is concordant with other studies likeOncu S. et als' study ${ }^{13}$.

Our date didn't show statistical relationship between length of hospitalization and the rate of infection but we should consider that we There was no statistical difference in positive catheter blood culture results based on the length of hospital stay, underlying disease, section hospitalized, catheter size number, and catheter insertion site.
Our study showed that underlying disease and the unit patients were admitted to didn't have any impact on the rate of infection which was similar to the study Oncu, et al conducted $^{13}$. Furthermore, studies were done by Sydman and et al., ${ }^{17}$, and Maki and et al., ${ }^{11}$ to compare the safe interval between changing sets of injection, come to the conclusion that the risk of complications with replacement every 72 hours compared.with replacement every 48 times of injection are not increasing .

Patients on chemotherapy agents,unlike those on antibiotics or corticosteroids, represented a significant statistical difference in the rate of positive blood culture $(\mathrm{p}=0.003)$. This means that the frequency of positive catheter blood culture in patients who had received chemotherapy was more than patients who had not received these drugs. Thus we can conclude that chemotherapy agent is a contributing factor to increase the rate of peripheral venous catheter induced blood infection.

Oncu's study conducted in Turkey showed the rate of catheter-related infections in patients who were on antibiotics glycopeptide's antibiotics was higher than the others ${ }^{13}$.

Staphylococcus aureus and coagulase negative Staphylococci were the most common organism isolated in our study. It is similar to the result of study was done by cultured positive in our study was, which concluded with the study of Oncu S. et al., ${ }^{13}$. However,Shukrallah B. et al., ${ }^{18}$ who reported that the most common catheterrelated infections were due to Staphylococcus coagulase negative and Staphylococcus aureus. InPujol M. et al. study ${ }^{14}$, Staph. aureus has been reported as the most common organism responsible for catheter induced blood infection.

ZahidNabi reported that coagulase negative staphyloccus, Staph. hemolyticus and Staph. Aureus were the most commoncausative organisms in their study ${ }^{13}$.

A study conducted by Iran University of Medical Sciences showed that prevention of HCRI (hemodialysis catheter related infections) requires the identification of predisposing risk factors. They studied 116 patients Pathogenic organisms isolated from blood cultures included Staphylococcus aureus 42\%, coagulase-negative staphylococci 20\%, E. coli 19\%, Enterococci 7\%, Streptococcus D 7\%, Pseudomonas aeruginosa 
4\%, and klebsiella 1\%. Bacterial resistance to vancomycin and amikacin was present in $7 \%$ and $4 \%$ of the cases, respectively. What sets these studies is clear that the most common cause of positive catheter blood culture results is grampositive cocci and Staphylococcus, as Staphylococcus aureus is involved more than S. epidermidis. They conclude that the prevalence of pathogenic organisms of HCRI were similar to previous studies ${ }^{19}$.

According to the above mentioned, the use of chemotherapy drugs is a risk factor for Catheter-related infection.

In conclusion, our study showed that the rate of catheter infection in our hospitals is higher than the national numbers so we need more studies to be done to recognize the reason for that. With more knowledge about the causes of our high rate of catheter infection, we will be able to implicate some preventive measures to improve patient care in our hospitals.

\section{REFERENCES}

1. Allon, M., Dialysis catheter-related bacteremia: treatment and prophylaxis. Am. J. Kidney Dis. 2004; 44: 779-91.

2. Band, D.J., Diagnosis and management of central venous catheter related infections.Up to Date 2005; 13.1 (800) 998-6374. (781) 237-4788.

3. Band, J.D.; Maki, D.G., Safety of changing intravenous delivery systems at longer than24hour intervals. Ann. Intern. Med. 1979; 91(2): 173-8.

4. Carson, L.A.; Favero, M.S.; Bond, W.W.; Petersen, N.J., Morphological, biochemical,and growth characteristics of pseudomonas cepacia from distilled water. Appl. Microbiol. 1973; 25(3): 476-83.

5. $\quad$ Farrington, K.; Rao, R; Gilg, J.; Ansell, D.; Feest, T., UK Renal Registry: New adultpatients starting renal replacement therapy in UK in 2005. UK Renal registry report.Bristol, UKchapter 2005; 3: 12-26.

6. Goldmann, D.A.; Martin, W.T.; Worthington, J.W., Growth of bacteria and fungi intotal parenteral nutrition solutions.Am. J. Surg. 1973; 126(3): 314-8.

7. Kozeny, G.A.; Venezio, F.R.; Bansal, V.K.; Vertuno, L.L.; Hano, J.E., Incidence ofsubclavian dialysis catheter-related infections. Arch. Intern. Med. 1984; 144: 1787-94.

8. Ljungman, P.; Hagglund, H.; Bjorkstrand, B.;
Lonnqvist, B.; Ringden, O., Preoperativeteicoplanin for prevention of grampositive infections in neutropenic patients with indwellingcentral venous catheters: a randomized, controlled studa. Support.Care.Cancer. 1997; 5(6): 485-8.

9. Maki, D.G., Epidemic nosocomial bacteremias. Am. J. Med. 1981; 70(3): 719-32.

10. Maki, D.G., Growth properties of microorganisms in infusion fluid and methods ofdetection. Microbiological hazards of infusion therapy. Springer Netherlands. 1976; ISBN 978 94-011-6179-4.p.13-74.

11. Maki, D.G.; Botticelli, J.T.; LeRoy, M.L.; Thielke, T.S., Prospective study ofreplacing administration sets for intravenous therapy at 48- vs 72-hour intervals. 72 hours is safeand cost-effective. JAMA. 1987; 258(13), 1777-81.

12. Nabi, Z.; Anwar, S.; Barhamein, M.; Al Mukdad, H.; El Nassri, A., Catheter related infection in hemodialysis patients. Saudi. J. Kidney. Dis. Transpl. 2009; 20(6): 1091-5.

13. Oncu, S.; Ozsut, H.; Yildirim, A.; Ay, P.; Cakar, N.; Eraksoy, H.; Calangu, S., Centralvenous catheter related infections: risk factors and the effect of glycopeptide antibiotics. Ann.Clin. Microbiol.Antimicrob. 2003; 27: 2:3.

14. Pujol, M.; Hornero, A.; Saballs, M.; Argerich, M.J.; Verdaguer, R.; Cisnal, M., Clinical epidemiology and outcomes of peripheral venous catheter-related bloodstream infections at a university-affiliated hospital. J. Hosp. Infect. 2007; 67(1): 22-9.

15. Rijnders, B.J.; Van Wijngaerden, E.; Vandecasteele, S.J.; Stas, M.; Peetermans, W.E., Treatment of long-term intravascular catheterrelated bacteraemia with antibiotic lock: randomized, placebo-controlled trial. $J$. Antimicrob. Chemother. 2005; 55(1): 90-4.

16. Salzman, M.B.; Rubin, L.G., Relevance of the catheter hub as portalfor microorganisms causing catheter-related bloodstream infections. Nutrition.1997; 13(4): 15S-7S.

17. Snydman, D.R.; Donnelly-Reidy, M.; Perry, L.K.; Martin, W.J., Intravenous tubing containing burettes can be safely changed at 72 hour intervals. Infect. Control. 1987; 8(3): 1136.

18. Shukrallah, B.; Hanna, H.; Hachem, R.; Ghannam, D.; Chatzinikolaou, I.; Raad, I., Correlation between early clinical response after catheter removal and diagnosis of catheterrelated bloodstream infection. Diagn. Microbiol. Infect. Dis. 2007; 58(4): 453-7.

19. Sanavi, S.; Ghods, A.; Afshar, R., Catheter associated infections in hemodialysis patients. 
Saudi. J. Kidney. Dis. Transpl. 2007; 18(1): 436.

20. Teehan, G.S.; Bahdouch, D.; Ruthazer, R., Iron storage indices: novel predictors of bacteremia in hemodialysis patients initiating intravenous iron therapy. Clin. Infect. Dis. 2004; 38: 1090 4.

21. Van de Wetering, M.D.; van Woensel, J.B.; Kremer, L.C.; Caron, H.N., Prophylactic antibiotics for preventing early Gram-positive central venous catheter infections in oncology patients, a Cochrane systematic review. Cancer. Treat. Rev. 2005; 31: 186-196.
.Harshal Shah, MBBS, 1 Wendelyn Bosch, MD,1 Kristine M. Thompson, MD,2 and Walter C. Hellinger, MD., Intravascular CatheterRelated Bloodstream InfectionNeurohospitalist. 2013; 3(3): 144-151 PMC3805442

23. Maki, DG, Mermel, LA. Infections due to infusion therapy. In: Hospital infections, Bennett JV, Brachman PS (Eds), Lippincott-Raven, Philadelphia, PA 1998; 689-724.

24. Issam Raad, Hend Hanna, Dennis Mak, Intravascular catheter-related infections: advances indiagnosis, prevention, and management. Lancet Infect Dis 2007; 7:645-57. 